\title{
The Relative Reinforcing Value of Sweet versus Savory Snack Foods after Consumption of Sugar- or Non-Nutritive Sweetened Beverages
}

Shanon L. Casperson, LuAnn Johnson, and James N. Roemmich

USDA, Agricultural Research Service, Grand Forks Human Nutrition Research Center, 2420 2nd Ave. North, Grand Forks, ND 58203-9034, USA

Address correspondence to: Shanon Casperson, Ph.D.

Research Biologist

USDA-ARS

Grand Forks Human Nutrition Research Center

2420 2nd Ave. North

Grand Forks, ND 58203-9034

Phone: 701-795-8497; Fax: 701-795-8230

Email: shanon.casperson@ars.usda.gov 


\begin{abstract}
The effects of sugar-sweetened (SSB) and non-nutritive sweetened (NSB) beverages on the regulation of appetite, energy intake and body weight regulation remain controversial. Using a behavioral choice paradigm, we sought to determine the effects of consuming a SSB or NSB on appetite and the reinforcing value of sweet relative to salty/savory snack foods. In a randomized crossover study, 21 healthy weight adults consumed $360 \mathrm{ml}$ of SSB (sucrose; $31 \mathrm{~g}$ ) or NSB (sucralose; 4g) with a standardized meal. Hedonic ratings for the sweet and salty/savory snack foods used for the reinforcement task were assessed prior to the start of the study. Satiety and the desire to eat foods with a specific taste profile were assessed before and every 30 minutes post-meal for $4 \mathrm{~h}$. The relative reinforcing value of the snack foods was assessed using a computer-based choice task (operant responding with concurrent schedules of reinforcement) $4 \mathrm{~h}$ post-meal. Hedonic ratings did not differ between the most highly liked sweet and salty/savory snack foods. Beverage type did not influence measures of satiety or the desire to eat foods with a specific taste. However, sweet snacks were more $(p<0.05)$ reinforcing relative to salty/savory snack foods after consuming a NSB than after a SSB. In conclusion, this is the first study to demonstrate that NSB can increase the motivation to gain access to sweet snacks relative to salty/savory snack foods later in the day.
\end{abstract}

\title{
Keywords
}

food reinforcement, sugar-sweetened, "diet" drinks, non-nutritive sweetened, snack foods 


\section{Introduction}

Sugar-sweetened beverages (SSBs) are the largest single source of added sugar and discretionary energy intake in the U.S. diet (Drewnowski \& Rehm, 2014). As such, a great deal of research has focused on their role in the etiology of obesity (see reviews (Kaiser, Shikany, Keating, \& Allison, 2013; Malik, Pan, Willett, \& Hu, 2013; Pereira, 2014)). Both clinical and observational studies demonstrate associations of SSB consumption with increased energy intake and body weight (Bachman, Baranowski, \& Nicklas, 2006; Bray \& Popkin, 2014; Hu, 2013; Kaiser, et al., 2013; Malik, et al., 2013; Malik, Schulze, \& Hu, 2006; Mattes, Shikany, Kaiser, \& Allison, 2011; Pereira, 2014). However, there are not yet definitive conclusions regarding the nature and strength of SSB consumption on appetite, energy intake and body weight regulation (Bachman, et al., 2006; Bray \& Popkin, 2014; Hu, 2013; Kaiser, et al., 2013; Malik, et al., 2013; Malik, et al., 2006; Mattes, et al., 2011; Pereira, 2014). Even so, there is a general consensus that the U. S. population would benefit from a reduction in added dietary sugars, including SSB intake (Health \& Services, 2015).

Non-nutritive sweeteners (NNS), also called low-calorie sweeteners (LCS) or sugar substitutes, were first introduced to the market as a specialty food for diabetics, but later gained mass appeal as a way to reduce energy intake (Yang, 2010). As with SSBs, there is continued debate about the effects of non-nutritive sweetened beverages (NSBs), which are the most common vehicles of NNS consumption (Mattes \& Popkin, 2009), on appetite, energy intake and body weight regulation (Peters \& Beck, 2016; Rogers, et al., 2016). It has been suggested that NSB consumption may increase energy intake via an uncoupling of sweet taste with its anticipated post-ingestive consequences (Burke \& Small, 2015). In animal models, NNS mixed into solid foods reduces the ability to use sweet taste to predict the energy content of the food (Rogers, et al., 2016; Swithers, 2016). In humans, habitual NSB consumption is associated with alterations in the reward experienced from both nutritive and nonnutritive sweet tastes (Green \& Murphy, 2012). Thus, one possible mechanism by which NNS, and NSB, consumption may lead to increased energy intake is by increasing the appetite for sweet tasting foods.

Another mechanism by which NSB or SSB may affect energy intake in humans is through changes in food choices by altering the reinforcing value of foods with specific taste profiles. Sugar and NNS affect central food reward areas that stimulate reward-driven eating behavior (Burke \& Small, 2015; Epstein, Carr, Lin, \& Fletcher, 2011; Sylvetsky, Rother, \& Brown, 2011; Yang, 2010), and sugar as a component of a food particularly increases the reinforcing value of that food (Avena, Rada, \& Hoebel, 2008; Epstein, et al., 2011). Food reinforcement motivates people to eat and predicts energy intake (Epstein, Leddy, Temple, \& Faith, 2007; Temple, 2014). Given the potential of NSB consumption to uncouple sweet taste and post-ingestive consequences (Burke \& Small, 2015) and that the sugar content of a food increases its 
reinforcing value (Epstein, et al., 2011), it can be posited that NNS, including those in NSBs, may increase the reinforcing value of sugar-rich sweet tasting foods later in the day in an attempt to recouple sweet taste with energy and post-ingestive consequences (Yang, 2010). However, the effect of NNS on the reinforcing value of foods with specific taste profiles is not yet known. The present study employed questionnaires and operant responding methods to test the effects of NSB consumption on later appetite and the reinforcing value of foods with sweet or salty/savory taste profiles.

\section{Methods}

\section{Participants}

Healthy weight adults (Table 1) were recruited from the greater Grand Forks, ND area. Screening for study eligibility included height, weight, taste testing of study foods, fasting glucose level (Accu-Chek), and a medical health history questionnaire. Exclusion criteria included: $\mathrm{BMI}>25 \mathrm{~kg} / \mathrm{m}^{2}$, allergies to any study foods, recent weight loss or gain, pregnancy, lactation, fasting glucose $>100$, active cancer or in short-term remission (less than 3 years), infectious diseases, alcohol or drug abuse, tobacco use, presence of acute illness, or taking medications known to affect energy expenditure and appetite. The study (clinicaltrials.gov as NCT02211599) was approved by the University of North Dakota Institutional Review Board and informed written consent was obtained from all participants prior to any study-related procedures.

Table 1: Participant characteristics

\begin{tabular}{|lc|}
\hline $\mathrm{N}(\mathrm{F} / \mathrm{M})$ & $21(11,10)$ \\
Age, yrs & $24 \pm 6$ \\
Height, cm & $174 \pm 11$ \\
Weight, kg & $69 \pm 14$ \\
BMI, kg/m² & $23 \pm 2$ \\
Body fat, \% & $19 \pm 7$ \\
Fat mass, kg & $16 \pm 11$ \\
Fat-free mass, kg & $55 \pm 18$ \\
\hline
\end{tabular}

Values are means \pm SD.

\section{Experimental Procedures}

Prior to testing each participant tasted and rated their liking on a 10-point scale of each food to be used for the food reinforcement task (Table 2). The evening before each testing session participants were admitted to the Metabolic Research Unit (MRU) at the Grand Forks Human Nutrition research Center (GFHNRC) and provided a standardized meal to control for nutrient intake. Participants completed 2 testing sessions separated by a minimum of 7 days and were 
instructed not to exercise for at least 48 hours prior to admission. The day of testing participants were provided a research specific breakfast at 08:00 and lunch at 12:00, and were required to consume all foods and beverages within 30 minutes. Meals provided 500 nonbeverage kcals and consisted of the same foods (potatoes, ham, cheddar cheese, white bread and butter). To minimize the monotony of consuming the same foods repeatedly for all meals, the foods were presented differently for breakfast and lunch. Breakfast was presented as a potato, cheese and ham casserole with a side of buttered toast and lunch was presented as a ham and cheese sandwich with a side of buttered potatoes. The amount of protein in the meals varied between the two visits. However, there was no effect of protein on the relative reinforcing value of sweet to salty/savory foods (primary outcome; $p=0.7$ ); therefore, protein was not included as a factor in the final analysis. The test beverage was presented with the lunch meal and was comprised of $360 \mathrm{~mL}$ of water, black cherry powdered drink mix, and either sugar (31g) or a non-nutritive sweetener (sucralose; $4 \mathrm{~g}$ ). Sucralose (Splenda) was matched to the sugar based on manufacturer conversions and blindly taste tested by study personnel to ensure that the same level of pleasantness and sweetness was achieved. The test beverage was presented to participants in a double-blind, randomized order to reduce cognitive bias. Satiety and the desire to consume foods with a specific taste profile was assessed before and every 30 minutes post-meal for a total of 4 hours. The reinforcing value of sweet snack foods relative to salty/savory snack foods was assessed 4 hours after lunch using a computer choice task (Epstein, et al., 2011). Participants received detailed instruction and were allowed a practice session. Water was provided ad libitum.

Table 2: Foods used for the food reinforcement task

\begin{tabular}{|lllllll|}
\hline & & \multicolumn{4}{c}{ Macronutrient breakdown per gram } \\
\cline { 2 - 7 } & & Kcal & CHO & Fat & Protein & Sugar \\
\hline \multirow{4}{*}{ Sweet snack foods } & Mini Oreos & 4.59 & 0.71 & 0.18 & 0.04 & 0.39 \\
\cline { 2 - 7 } & M\&Ms & 4.94 & 0.71 & 0.21 & 0.04 & 0.63 \\
\cline { 2 - 7 } & Skittles & 4.03 & 0.93 & 0.03 & 0.00 & 0.76 \\
\cline { 2 - 7 } & Reese's Pieces & 5.00 & 0.63 & 0.26 & 0.07 & 0.53 \\
\hline \multirow{3}{*}{$\begin{array}{l}\text { Salty/Savory snack } \\
\text { foods }\end{array}$} & Doritos & 4.94 & 0.57 & 0.29 & 0.07 & 0.00 \\
\cline { 2 - 7 } & Cheez-Its & 5.00 & 0.63 & 0.27 & 0.10 & 0.00 \\
\cline { 2 - 7 } & Pretzels & 3.88 & 0.81 & 0.00 & 0.04 & 0.02 \\
\cline { 2 - 7 } & Pringles & 5.26 & 0.58 & 0.32 & 0.04 & 0.00 \\
\hline
\end{tabular}

\section{Food reinforcement task}

The relative reinforcing value (RRV) of sweet and salty/savory snack foods was assessed via a computer game that requires operant responding. Operant responding is a classical means of measuring the reinforcing value of rewards such as a sugary or savory snack (Epstein, et al., 2007). Two separate computer workstations made up the experimental environment. At one 
station the participant could complete button presses to earn their most liked sweet snack food and at the other station they could work for their most liked salty/savory snack food.

Participants could work at their pace and move freely back and forth between the computer workstations. The computer program mimics a slot machine and points are earned each time 3 matching shapes appear. For each 5 points earned, participants received a small portion ( 14 $24 \mathrm{~g}$ ) of the food reward they were working toward. The work to gain access to each snack food increased on independent and concurrent $\log _{2}(x)$ variable reinforcement schedules $( \pm 25 \%)$ beginning at 4 clicks per point.

The session ended when the participant no longer wanted to earn points. Participants were monitored at all times during testing. The breakpoint ( $P_{\max }$ last schedule completed) and the total number of responses ( $\left.P_{\text {response }}\right)$ performed were recorded for each alternative. The proportion of breakpoint ( $R R V P_{\max }$ ) and the proportion of responses (RRV $P_{\text {response }}$ ) for the sweet snack food was compared with that of the salty/savory snack food and was calculated as

RRV $P_{\max }=P_{\max \text { for sweet }} /\left(P_{\max \text { for sweet }}+P_{\max \text { for salty/savory }}\right)$

RRV $P_{\text {response }}=P_{\text {response for sweet }} /\left(P_{\text {response for sweet }}+P_{\text {response for salty } / \text { savory }}\right)$.

The breakpoint, total responses, and RRV were calculated and used as metrics of reinforcement (Epstein, Carr, Lin, Fletcher, \& Roemmich, 2012).

Earned portions of the energy-dense snack foods were provided to the participant after they

indicated that they had finished the task. Participants were instructed that they did not have to consume all of the food that they earned and that they would not be able to take the food out of the laboratory. Participants were informed that if they felt that they needed more food than they had originally earned they could resume playing. Any uneaten portions of the snack foods were sent back to the metabolic kitchen to be weighed and recorded. One participant (male) resumed playing for more snacks. During the exit interview the participant stated that he wanted a little more food than he had originally earned.

\section{Homeostatic and hedonic hunger}

Subjective homeostatic and hedonic hunger ratings were assessed using a computer-based visual analogue scale (Sussex Ingestion Pattern Monitor, University of Sussex). Participant's subjective rating of hunger, fullness, satiation, desire to eat something fatty, salty, sweet or savory, and prospective food consumption was recorded immediately before the lunch meal and then every 30 minutes for a total of 4 hours. Questions asked included: 1 . How hungry do you feel; 2 . How strong is your desire to eat; 3. How full do you feel; 4 . How satisfied do you feel; 5 . Would you like to eat something sweet; 6 . Would you like to eat something salty; 7 . Would you like to eat something savory; 8 . Would you like to eat something fatty. Each 
question appeared on the monitor with a vertical bar and horizontal line below each question. Using the mouse, participants moved the vertical bar to the position on the horizontal line that best described their rating for the current question. All ratings were scored automatically on a scale from 0 (not at all) to 100 (very much).

\section{Statistical analysis}

The primary outcome was the RRV of sweet to salty/savory foods. The homeostatic and hedonic hunger ratings for each participant and beverage type were summarized by calculating the area under the curve (AUC) using the trapezoid rule. The effects of beverage type on AUC subjective homeostatic and hedonic hunger ratings, RRV of sweet to salty/savory foods, and amounts of snacks earned and consumed during the food reinforcement task were assessed using a mixed model analysis of variance in which beverage type was the fixed effect and participant was treated as a random effect. $P_{\max }$ and $P_{\text {response }}$ were analyzed using a mixed model analysis of variance with beverage type and snack preference (sweet or salty/savory) and their interactions included as fixed effects and participants as random effects. $P_{\text {response }}$ was $I n-$ transformed prior to analysis to reduce skewness. The difference between the amounts of snacks earned and consumed during the food reinforcement task were analyzed using a mixed model analysis of variance in which participant and visit were random effects. SAS V9.4 (SAS Institute, Inc., Cary, NC) was used for all analyses. Data are reported as means \pm standard error of the mean (SE). 


\section{Results}

Subjective appetite responses

Hunger and satiety scores are shown in Figure 1. There was no difference in the AUC for the 4 satiety questions between the SSB and NSB conditions.

A.

How hungry do you feel?

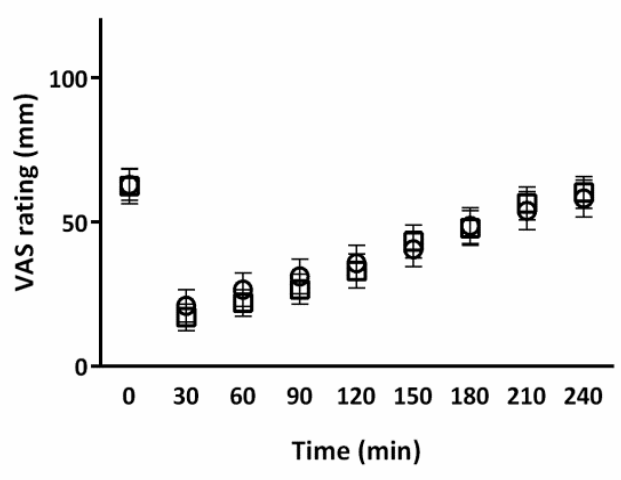

C.

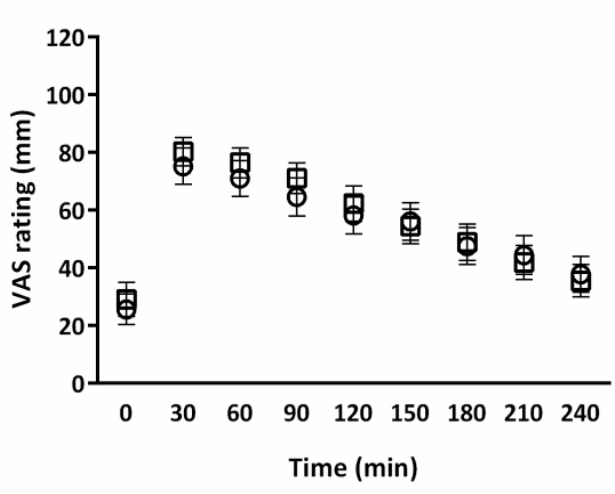

B. How strong is your desire to eat?

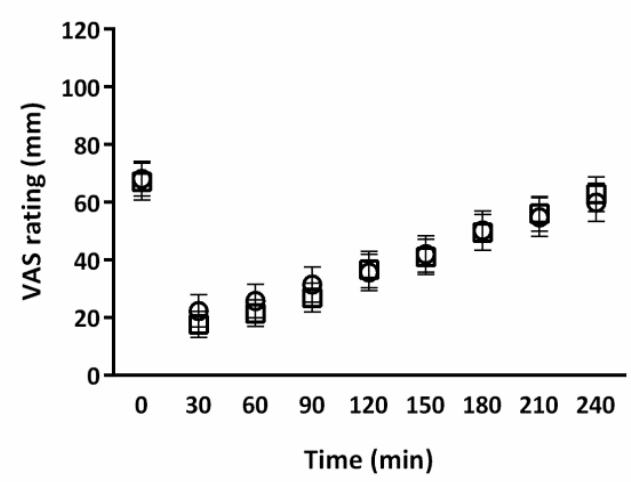

D. How satisfied do you feel?

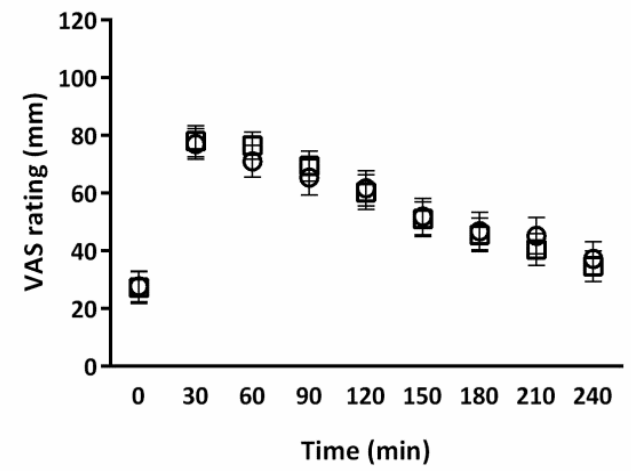

Figure 1: Basal (time 0) and postprandial subjective satiety scores for (A) hunger, (B) desire to eat, (C) fullness, and (D) satiation in response to the inclusion of a sugar- (open squares) or non-nutritive- (open circles) sweetened beverage with a standardized meal. Data are presented as means $\pm \mathrm{SE}$. 
Ratings for the desire to eat something sweet, salty, savory and fatty are shown in Figure $\mathbf{2}$. There was no difference in the AUC between the SSB and NSB conditions.

A. Would you like to eat something sweet?

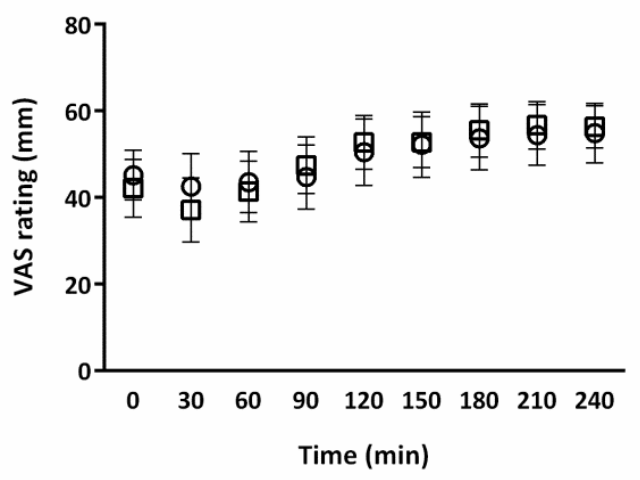

C. Would you like to eat something savory?

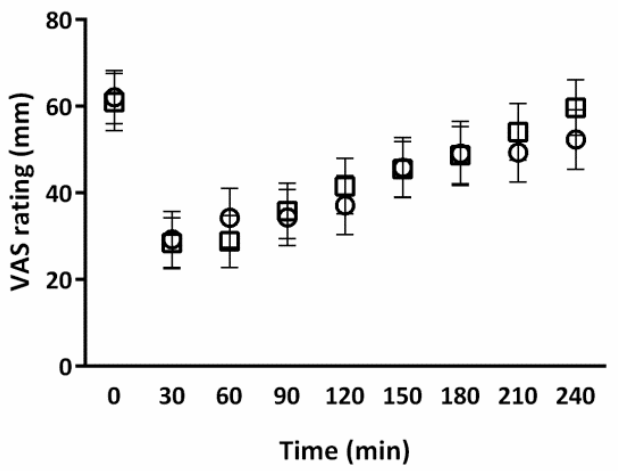

B. Would you like to eat something salty?

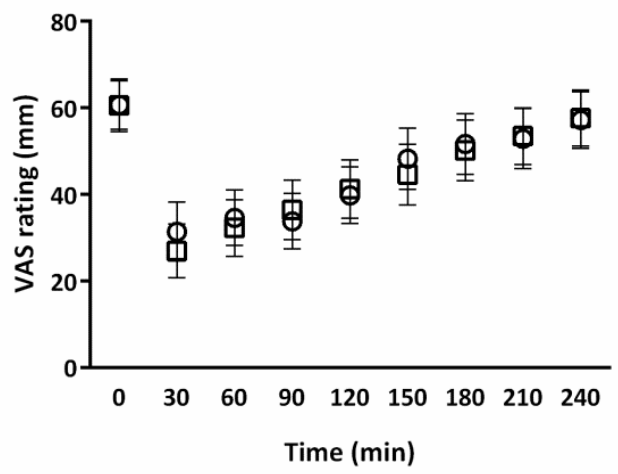

D. Would you like to eat something fatty?

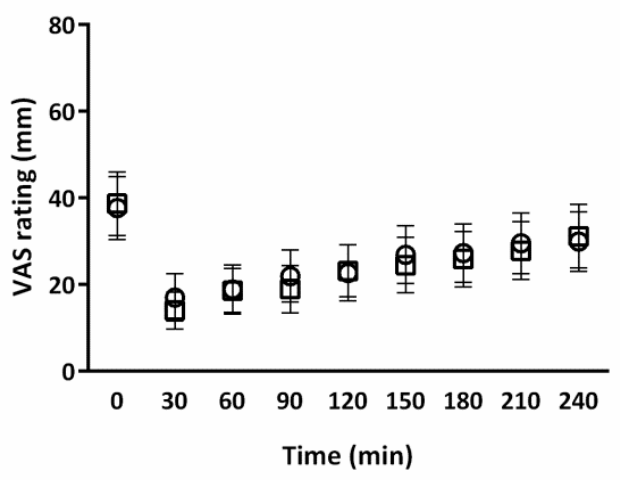

Figure 2: Basal (time 0) and postprandial appetite scores for the desire to eat something (A) sweet, (B) salty, (C) savory, and (D) fatty foods in response to the inclusion of a sugar- (open squares) non-nutritive (open circles) sweetened beverage with a standardized meal. Data are presented as means $\pm \mathrm{SE}$.

Hedonic and motivational ratings of snack foods

There was no difference in VAS measures of liking of the sweet $(9 \pm 1)$ and savory $(9 \pm 1)$ snack foods used for the food reinforcement task. When SSB was consumed with lunch, participants performed $294 \pm 77$ responses with a breakpoint of $3 \pm 0.3$ schedules completed for the sweet snack food and $562 \pm 170$ responses with a breakpoint of $3 \pm 0.5$ schedules completed for salty/savory snack food of their choice. When participants consumed the NSB with their lunch meal they performed $434 \pm 96$ responses with a breakpoint of $4 \pm 0.4$ for their sweet snack food choice and $329 \pm 118$ responses with a breakpoint of $3 \pm 0.4$ for their salty/savory snack food choice. 
There was a significant effect of the beverage sweetener type on RRV $P_{\max }$ and RRV $P_{\text {responses }}$ of sweet to salty/savory snack foods ( $p<0.03$ and $p<0.04$, respectively; Figure 3 ). The consumption of NSB increased RRV of sweet snack foods (i.e., candy) to salty/savory snack foods (i.e. chips).

A.

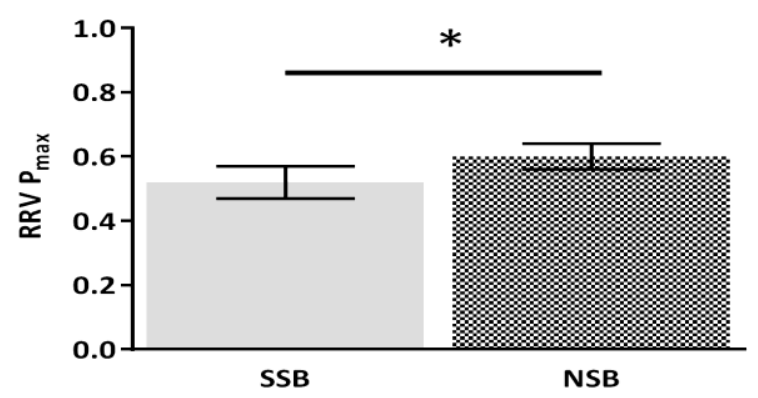

B.

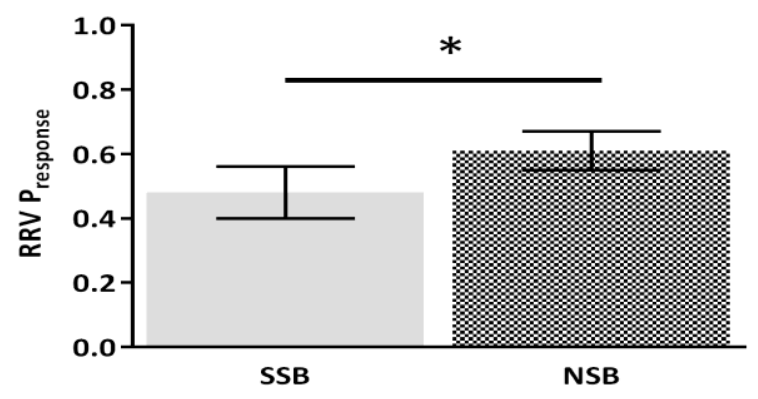

Figure 3: Relative reinforcing value (RRV) of sweet to salty/savory snack foods when measured as $P_{\max }$ (upper panel) and $\mathrm{P}_{\text {response }}$ (lower panel) 4 hours after the consumption of a sugar- or non-nutritive sweetened beverage (SSB and NSB, respective) with a standardized meal. Values are expressed as means \pm SE. * significant difference between the amount of work performed to gain access to the sweet compared to the salty/savory snack foods.

\section{Energy-dense snack food intake}

There was a main effect of the snack food type on the amount of energy earned during the RRV task $(p=0.01)$. Overall, more of the sweet snack foods were earned compared to the salty/savory snack foods. After NSB consumption $294 \pm 63 \mathrm{kcal}$ of the sweet snack food and 205 \pm 44 kcals of the salty/savory snack food were earned. After SSB consumption $283 \pm 37 \mathrm{kcal}$ of the sweet snack food and $249 \pm 41$ kcals of the salty/savory snack food were earned. There was no interaction between the type of sweetener in the beverage and the amount of sweet and salty/savory snacks foods earned. 
There was a main effect of the snack food type on the amount of energy consumed after the RRV task $(p=0.01)$. Overall, more of the sweet snack foods were consumed compared to the salty/savory snack foods. When NSB was consumed with lunch $252 \pm 54 \mathrm{kcal}$ of the sweet snack food and $168 \pm 36$ kcals of the salty/savory snack food were consumed. After SSB consumption $238 \pm 40 \mathrm{kcal}$ of the sweet snack food and $219 \pm 36 \mathrm{kcals}$ of the salty/savory snack food were consumed. There was a trend $(p=0.08)$ for an interaction between the type of sweetener in the beverage and the amount of sweet and salty/savory snacks foods consumed with more of sweet snack foods being consumed after the NSB.

There was no effect of beverage type on the grams of sugar earned $(p=0.13)$ or consumed $(p=$ $0.11)$. When NSB was consumed with lunch $38 \pm 33 \mathrm{~g}$ of sugar was earned, of which participants consumed $34 \pm 32 \mathrm{~g}$. After SSB consumption $40 \pm 30 \mathrm{~g}$ of the sugar was earned and $34 \pm 30 \mathrm{~g}$ was consumed.

Overall, participants consumed less of the sweet snack foods than they earned during the RRV task $(p=0.03)$. There was a trend $(p=0.06)$ toward less of salty/savory snack foods being consumed than earned. There was no main effect of beverage sweetener type on the total amount of energy earned (data not shown) or consumed from the sweet snack foods or the salty/savory snack foods (Figure 4).

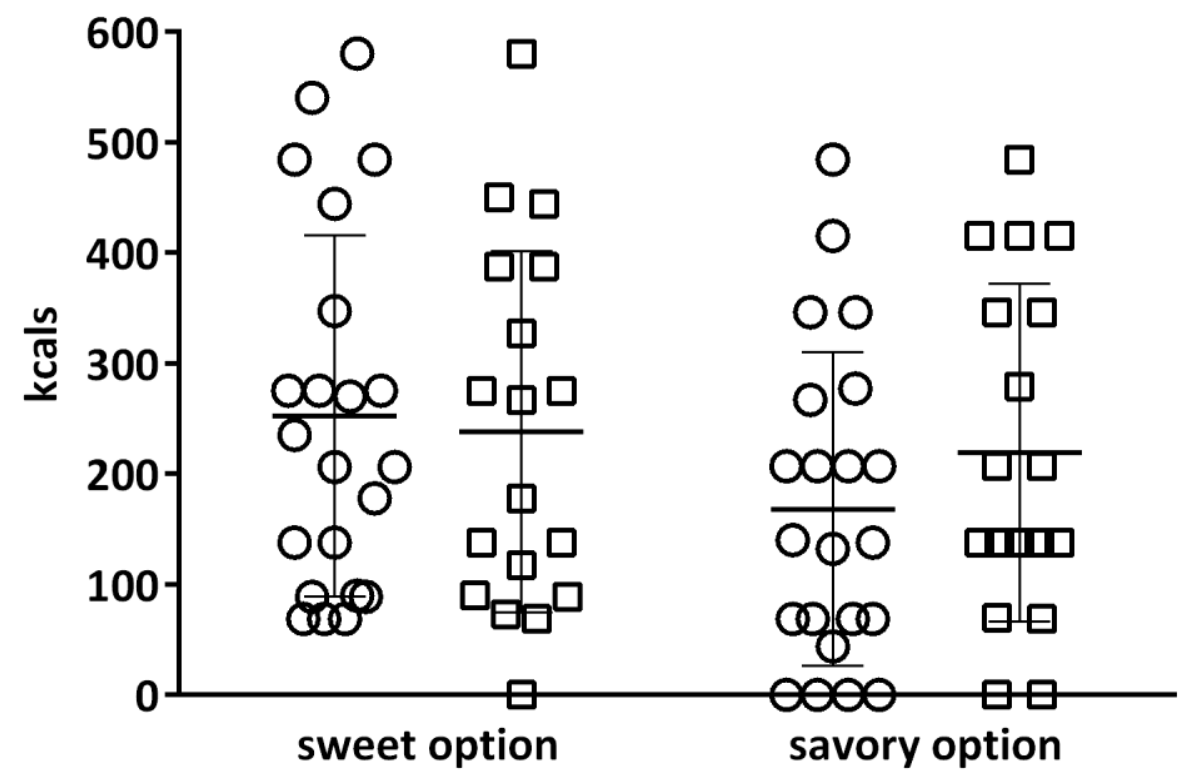

Figure 4: Energy (Calories) consumed from the sweet and salty/savory snack foods earned during the food reinforcement task. Total energy consumed by each participant after drinking the sugar-sweetened beverage (SSB), represented by the open squares, and the non-nutritive sweetened beverage (NSB), 210 represented by the open circles. Lines represent the mean \pm SD. 


\section{Discussion}

The present study demonstrates for the first time that the type of sweetener in a beverage consumed with the lunch meal influences the reinforcing value of sweet relative to salty/savory snack foods. In this sample of healthy adults the consumption of a NSB, compared to a SSB, increased the RRV of the sweet snack foods. That is, 4 hours after consuming a NSB at lunch, the participants were willing to do more work to gain access to a sweet snack than a salty/savory snack. In contrast, Birch and colleagues (Birch, McPhee, \& Sullivan, 1989) found that children's test meal food choices after NSB and SSB consumption depended on individual preferences for the foods not on the taste profile of the foods.

Though there was an increase in the RRV of sweet snack foods and sweet snack food calories after consumption of NSB, measures of appetite or total snack food energy intake did not differ between the NSB and SSB conditions. These results support previous reports that NNS increases subjective measures of the motivation to eat without an increase in test meal energy intake (Rogers, Carlyle, Hill, \& Blundell, 1988). The RRV of food is associated with energy intake immediately after completing the task and with usual energy intake (Epstein, et al., 2011). Thus, it seems that NSB consumption may uncouple the relationship between the motivation for a sweet food and eating behavior, at least temporarily. These results build upon the knowledge provided by functional magnetic resonance imaging (fMRI) studies on neurobehavioral pathways regulating eating behavior. Researchers using fMRI have shown that even though sugar stimulates more regions in the brain related to food reward compared to NNS (sucralose); the connectivity within the food reward pathways is stronger with NNS consumption (Frank, et al., 2008). Because NNS is not accompanied by the same post-ingestive consequences as sucrose, these neural response differences may explain the greater motivation to gain access to sweet tasting snack foods observed in this study. A notable finding from the current study is that all participants earned and consumed at least one portion of their most liked sweet snack food after the NSB, whereas this was not the case after SSB consumption. Thus, the data presented here support the hypothesis of a possible uncoupling between the sweet taste provided by artificial sweeteners and increased desire to consume sweet tasting foods (Yang, 2010).

The effects of NNS on appetite and energy intake appear to be dependent upon the conditions under which it is consumed. The augmentation of appetite appears to be influenced by the amount of energy consumed with the NNS (Mattes \& Popkin, 2009; Peters \& Beck, 2016; Rogers, et al., 2016) and perhaps the amount of time between NSB consumption and the test meal (Lavin, French, \& Read, 1997). When consumed alone in liquid form, NNS stimulates appetite (Lavin, et al., 1997; Rogers, et al., 1988). In fact, Lavin and colleagues (Lavin, et al., 1997) demonstrated a long-delay in the effect of NSB consumption on eating behavior, with 
increases in energy intake, predominately as carbohydrate, the day after consuming an aspartame-sweetened beverage. However, when NNS is consumed the same time as other foods stimulation of appetite sensations appears to be abolished (see review (Mattes \& Popkin, 2009)). Consistent with these results, the current study did not observe a difference between the inclusion of a SSB or a NSB with a mixed meal on subjective appetite sensations. Congruently, no difference was found in energy intakes of both the sweet and salty/savory snack foods in response to SSB and NSB consumption. These results are supported by recent animal data (Boakes, Kendig, Martire, \& Rooney, 2016) and analysis of National Health and Nutrition Examination Surveys data demonstrating similar energy intakes from sweet and salty/savory snack foods between healthy weight NSB and SSB consumers (Bleich, Wolfson, Vine, \& Wang, 2014).

This study is not without limitations. First, only healthy weight adults were recruited for participation. Weight status influences the RRV of food (Epstein, et al., 2007) and energy intake from discretionary foods (An, 2016; Bleich, et al., 2014). Therefore, it is possible that overweight and obese individuals may exhibit different appetitive or motivational responses in response to NSB consumption. However, the current study of healthy weight adults provides a basic indication of how NSB consumption at a meal can increase motivation for, and consumption of, sweet-tasting foods later in the day. Second, only a limited number of energydense snack foods were studied. It is possible that the RRV of snack foods with much different macronutrient compositions than those tested here would have produced different results. Still, it is intriguing that NSB increased the RRV of foods very high in sugar. Third, we studied only one type of artificial sweetener and did not include a water control. Our decision to not use a water control and to only use sucralose was to ensure that participants and study personnel were blinded. Many of the artificial sweeteners on the market have distinct, easily detected aftertastes, the exception is sucralose (Schiffman, Booth, Losee, Pecore, \& Warwick, 1995). Further studies are needed to determine whether different artificial sweeteners alter the relative reinforcing value of energy-dense snack foods in a similar manner. Additionally, even though NSBs are typically consumed with a meal (Mattes \& Popkin, 2009), consumption of these types of beverages without the added energy intake from solid foods may produce different effects on the RRV of energy-dense snack foods. Finally, the complex macronutrient composition of the energy-dense snack foods used for this study only allow for the conclusion of differences in the RRV of sweet and salty/savory tasting foods. We did, however, control for added sugar in the salty/savory food options.

In conclusion, these results reveal for the first time that consuming a NSB with a meal influences the RRV of energy-dense sweet snack foods. These results also demonstrate a possible short-term effect of NSB consumption on the uncoupling of the appetite for foods with sweet taste and the objective motivation to gain access to sweet foods. Taken together, these 
results suggest that NSBs, specifically those sweetened with sucralose, may play a role in altering eating behavior and food choices. The results of this study are important given that snacking accounts for $24 \%$ of daily energy intake and approximately $80 \%$ of the adult population consumes 2 or more snack servings each day ("Food Surveys : WWEIA Data Tables," 2015). Understanding the impact that beverages have on subsequent food choices is important for the development of innovative methods to alter eating behavior for the treatment and/or prevention of obesity and to inform public health recommendations.

\section{Acknowledgements}

The authors would like to thank Clint Hall, Doreen Rolshoven, Jackie Nelson and the GFHNRC dieticians and kitchen staff for their assistance with implementation of the protocol, data collection and data entry.

Funding: This work was supported by the Agricultural Research Services of the United States Department of Agriculture \#5450-51530-051-00D. The role of the finding sponsor was to approve the study and the submission of this manuscript for publication.

Disclosure Statement: The authors have nothing to disclose. Mention of trade names or commercial products in this publication is solely for the purpose of providing specific information and does not imply recommendation or endorsement by the U.S. Department of Agriculture. The U.S. Department of Agriculture (USDA) prohibits discrimination in all its programs and activities on the basis of race, color, national origin, age, disability, and where applicable, sex, marital status, familial status, parental status, religion, sexual orientation, genetic information, political beliefs, reprisal, or because all or part of an individual's income is derived from any public assistance program. (Not all prohibited bases apply to all programs.) Persons with disabilities who require alternative means for communication of program information (Braille, large print, audiotape, etc.) should contact USDA's TARGET Center at (202) 720-2600 (voice and TDD). To file a complaint of discrimination, write to USDA, Director, Office of Civil Rights, 1400 Independence Avenue, S.W., Washington, D.C. 20250-9410, or call (800) 795-3272 (voice) or (202) 720-6382 (TDD). USDA is an equal opportunity provider and employer. 


\section{References}

An, R. (2016). Beverage Consumption in Relation to Discretionary Food Intake and Diet Quality among US Adults, 2003 to 2012. J Acad Nutr Diet, 116, 28-37.

Avena, N. M., Rada, P., \& Hoebel, B. G. (2008). Evidence for sugar addiction: behavioral and neurochemical effects of intermittent, excessive sugar intake. Neurosci Biobehav Rev, 32, 20-39.

Bachman, C. M., Baranowski, T., \& Nicklas, T. A. (2006). Is there an association between sweetened beverages and adiposity? Nutr Rev, 64, 153-174.

Bleich, S. N., Wolfson, J. A., Vine, S., \& Wang, Y. C. (2014). Diet-beverage consumption and caloric intake among US adults, overall and by body weight. Am J Public Health, 104, e72-78.

Boakes, R. A., Kendig, M. D., Martire, S. I., \& Rooney, K. B. (2016). Sweetening yoghurt with glucose, but not with saccharin, promotes weight gain and increased fat pad mass in rats. Appetite, $105,114-$ 128.

Bray, G. A., \& Popkin, B. M. (2014). Dietary sugar and body weight: have we reached a crisis in the epidemic of obesity and diabetes?: health be damned! Pour on the sugar. Diabetes Care, 37, 950-956.

Burke, M. V., \& Small, D. M. (2015). Physiological mechanisms by which non-nutritive sweeteners may impact body weight and metabolism. Physiol Behav, 152, 381-388.

Drewnowski, A., \& Rehm, C. D. (2014). Consumption of added sugars among US children and adults by food purchase location and food source. Am J Clin Nutr, 100, 901-907.

Epstein, L. H., Carr, K. A., Lin, H., \& Fletcher, K. D. (2011). Food reinforcement, energy intake, and macronutrient choice. Am J Clin Nutr, 94, 12-18.

Epstein, L. H., Carr, K. A., Lin, H., Fletcher, K. D., \& Roemmich, J. N. (2012). Usual energy intake mediates the relationship between food reinforcement and BMI. Obesity (Silver Spring), 20, 1815-1819.

Epstein, L. H., Leddy, J. J., Temple, J. L., \& Faith, M. S. (2007). Food reinforcement and eating: a multilevel analysis. Psychol Bull, 133, 884-906.

Frank, G. K., Oberndorfer, T. A., Simmons, A. N., Paulus, M. P., Fudge, J. L., Yang, T. T., \& Kaye, W. H. (2008). Sucrose activates human taste pathways differently from artificial sweetener. Neuroimage, 39, 1559-1569.

Green, E., \& Murphy, C. (2012). Altered processing of sweet taste in the brain of diet soda drinkers. Physiol Behav, 107, 560-567.

Health, U. D. O., \& Services, H. (2015). 2015-2020 Dietary Guidelines for Americans. In: US Government Printing Office Washington, DC.

Hu, F. B. (2013). Resolved: there is sufficient scientific evidence that decreasing sugar-sweetened beverage consumption will reduce the prevalence of obesity and obesity-related diseases. Obes Rev, 14, 606-619.

Kaiser, K. A., Shikany, J. M., Keating, K. D., \& Allison, D. B. (2013). Will reducing sugar-sweetened beverage consumption reduce obesity? Evidence supporting conjecture is strong, but evidence when testing effect is weak. Obesity Reviews, 14, 620-633.

Lavin, J. H., French, S. J., \& Read, N. W. (1997). The effect of sucrose- and aspartame-sweetened drinks on energy intake, hunger and food choice of female, moderately restrained eaters. Int $J$ Obes Relat Metab Disord, 21, 37-42.

Malik, V. S., Pan, A., Willett, W. C., \& Hu, F. B. (2013). Sugar-sweetened beverages and weight gain in children and adults: a systematic review and meta-analysis. Am J Clin Nutr, 98, 1084-1102.

Malik, V. S., Schulze, M. B., \& Hu, F. B. (2006). Intake of sugar-sweetened beverages and weight gain: a systematic review. Am J Clin Nutr, 84, 274-288.

Mattes, R. D., \& Popkin, B. M. (2009). Nonnutritive sweetener consumption in humans: effects on appetite and food intake and their putative mechanisms. Am J Clin Nutr, 89, 1-14. 
Mattes, R. D., Shikany, J. M., Kaiser, K. A., \& Allison, D. B. (2011). Nutritively sweetened beverage consumption and body weight: a systematic review and meta-analysis of randomized experiments. Obes Rev, 12, 346-365.

Pereira, M. A. (2014). Sugar-sweetened and artificially-sweetened beverages in relation to obesity risk. Adv Nutr, 5, 797-808.

Peters, J. C., \& Beck, J. (2016). Low Calorie Sweetener (LCS) use and energy balance. Physiol Behav.

Rogers, P. J., Carlyle, J. A., Hill, A. J., \& Blundell, J. E. (1988). Uncoupling sweet taste and calories: comparison of the effects of glucose and three intense sweeteners on hunger and food intake. Physiol Behav, 43, 547-552.

Rogers, P. J., Hogenkamp, P. S., de Graaf, C., Higgs, S., Lluch, A., Ness, A. R., Penfold, C., Perry, R., Putz, P., Yeomans, M. R., \& Mela, D. J. (2016). Does low-energy sweetener consumption affect energy intake and body weight? A systematic review, including meta-analyses, of the evidence from human and animal studies. Int J Obes (Lond), 40, 381-394.

Schiffman, S. S., Booth, B. J., Losee, M. L., Pecore, S. D., \& Warwick, Z. S. (1995). Bitterness of sweeteners as a function of concentration. Brain Res Bull, 36, 505-513.

Swithers, S. E. (2016). Not-so-healthy sugar substitutes? Curr Opin Behav Sci, 9, 106-110.

Sylvetsky, A., Rother, K. I., \& Brown, R. (2011). Artificial sweetener use among children: epidemiology, recommendations, metabolic outcomes, and future directions. Pediatr Clin North Am, 58, 1467$1480, x i$.

Temple, J. L. (2014). Factors that influence the reinforcing value of foods and beverages. Physiol Behav, 136, 97-103.

. What We Eat in America, NHANES 2011-2012. In. (2015): USDA Agricultural Research Service.

Yang, Q. (2010). Gain weight by "going diet?" Artificial sweeteners and the neurobiology of sugar cravings: Neuroscience 2010. Yale J Biol Med, 83, 101-108. 